TRANSACTIONS OF THE

AMERICAN MATHEMATICAL SOCIETY

Volume 364, Number 7, July 2012, Pages 3427-3447

S 0002-9947(2012)05375-X

Article electronically published on March 8, 2012

\title{
CONVEX HYPERSURFACES WITH PINCHED PRINCIPAL CURVATURES AND FLOW OF CONVEX HYPERSURFACES BY HIGH POWERS OF CURVATURE
}

\author{
BEN ANDREWS AND JAMES MCCOY
}

\begin{abstract}
We consider convex hypersurfaces for which the ratio of principal curvatures at each point is bounded by a function of the maximum principal curvature with limit 1 at infinity. We prove that the ratio of the circumradius to the inradius is bounded by a function of the circumradius with limit 1 at zero. We apply this result to the motion of hypersurfaces by arbitrary speeds which are smooth homogeneous functions of the principal curvatures of degree greater than one. For smooth, strictly convex initial hypersurfaces with ratio of principal curvatures sufficiently close to one at each point, we prove that solutions remain smooth and strictly convex and become spherical in shape while contracting to points in finite time.
\end{abstract}

\section{INTRODUCTION}

Recently several papers have considered the flow of convex hypersurfaces by speeds which are homogeneous functions of the principal curvatures of degree $\alpha>1$. Under suitable pinching conditions on the curvature of the initial hypersurface, the aim is to prove that solutions become spherical as they contract to points. This behaviour has been established for a wide range of flows where the speed is homogeneous of degree 1 in the principal curvatures, including flow by the mean curvature [H1, the $n$th root of Gauss curvature Ch1, the square root of scalar curvature [Ch2], and a large family of other speeds [A1, A3, A4]. The first such result with degree of homogeneity higher than 1 was due to Ben Chow [Ch1], and concerned flow by powers of the Gauss curvature. He proved that flow by $K^{\beta}$ with $\beta \geq 1 / n$ produces a spherical limiting shape provided the initial hypersurface is sufficiently pinched, in the sense that $h_{i j} \geq C(\beta) H g_{i j}$. Later such results were proved by Schulze [S3] for powers of the mean curvature, by Alessandroni and Sinestrari for powers of the scalar curvature [A, AS, and by Cabezas-Rivas and Sinestrari CRS for normalized flows by powers of elementary symmetric functions.

A feature of the results mentioned above is that the flows all have some divergence structure, a point which is used crucially in deriving sufficient regularity of solutions to deduce the existence of a smooth limiting hypersurface. In [Ch1 the divergence structure was used to deduce pinching of the principal curvatures using integral

Received by the editors October 1, 2009 and, in revised form, April 28, 2010.

2010 Mathematics Subject Classification. Primary 53C44, 35K55; Secondary 53C40, 53C21, $58 \mathrm{~J} 35$.

This research was partially supported by Discovery Grant DP0556211 of the Australian Research Council and by a University of Wollongong Research Council small grant entitled "Flow of convex hypersurfaces to spheres by high powers of curvature".

(C)2012 American Mathematical Society 
estimates in a manner similar to [H1]. In [S3], $[\mathrm{A}$, and $[\mathrm{AS}]$ the curvature pinching was proved using maximum principle arguments, but the divergence structure was still needed because higher regularity of solutions was established using results for divergence form porous-medium equations.

In this paper we provide a geometric estimate which circumvents this difficulty. We prove in Section 3 that if the ratios of principal curvatures at each point are bounded in terms of the maximum principal curvature by a function which approaches one at infinity, then the ratio of the circumradius to the inradius is as close to one as desired if the circumradius is sufficiently small. Using this, together with a simple argument using spherical barriers, we deduce a positive lower bound on the speed for solutions of the flow equations, at times sufficiently close to the final time. This enables us to prove a result for flows with no special divergence structure. Somewhat surprisingly, we are able to prove the result for arbitrary smooth speeds which are strictly parabolic and homogeneous of degree $\alpha>1$, provided the pinching ratio of the initial hypersurface is sufficiently close to 1 depending only on $n, \alpha$ and a bound for the second derivatives of the speed. In particular we make no assumptions involving convexity or concavity of the speed as a function of the principal curvatures.

We note that the first author considered flows of surfaces in three-dimensional space by quite general functions of curvature A4, and the present paper provides the results required to prove smooth convergence to spheres for all the flows considered in that paper.

There are a few results that give a successful treatment of particular high order flows, requiring only (strict) convexity rather than any pinching condition. The first author proved such a result for Gauss curvature flow of a convex surface in threedimensional space [A2], and Schulze and Schnürer [S3] treated flows of surfaces by powers of mean curvature between 1 and 5 . Schnürer [S2] treated flow of surfaces with speed $\|A\|^{2}$, as well as a collection of other particular flows. While the results of the present paper show that sufficiently strong pinching is preserved by very general flows, understanding the behaviour of such flows without such a pinching requirement seems to be a much more subtle and difficult problem.

\section{NotATION AND PRELIMINARY RESUlts}

We denote the principal curvatures of a hypersurface $M$ by $\kappa_{\min }=\kappa_{1} \leq \cdots \leq$ $\kappa_{n}=\kappa_{\text {max }}$. These are the eigenvalues of the second fundamental form $A=\left(h_{i j}\right)$ with respect to the metric $\left(g_{i j}\right)$. The trace of $A$ with respect to $g$ is the mean curvature $H=\kappa_{1}+\cdots+\kappa_{n}$, while $\|A\|^{2}$ denotes the squared norm

$$
\|A\|^{2}=g^{i j} g^{k l} h_{i k} h_{j l}=\kappa_{1}^{2}+\cdots+\kappa_{n}^{2} .
$$

The trace free second fundamental form $\AA$ has components $h_{i j}-\frac{1}{n} H g_{i j}$. The length of $\AA$ satisfies

$$
\|\AA\|^{2}=\|A\|^{2}-\frac{1}{n} H^{2}=\frac{1}{n} \sum_{i<j}\left(\kappa_{i}-\kappa_{j}\right)^{2},
$$

which is a measure of the differences between the principal curvatures. We also make use of the elementary symmetric functions of curvature, defined by

$$
E_{k}=\frac{1}{\left(\begin{array}{l}
n \\
k
\end{array}\right)} \sum_{1 \leq i_{1}<\cdots<i_{k} \leq n} \kappa_{i_{1}} \ldots \kappa_{i_{k}}
$$


In our analysis of the flow equations we will use several geometric estimates for hypersurfaces. The first of these appears in [H1].

\section{Lemma 2.1.}

$$
\|\nabla A\|^{2} \geq \frac{3}{n+2}\|\nabla H\|^{2}
$$

and equivalently

$$
\|\nabla \AA\|^{2}=\left\|\nabla_{i} h_{j k}-\frac{1}{n} g_{j k} \nabla_{i} H\right\|^{2} \geq \frac{2(n-1)}{3 n}\|\nabla A\|^{2} .
$$

The next result gives a bound on the ratio of principal curvatures if the length of the traceless second fundamental form is small enough compared to the mean curvature:

Lemma 2.2. If $H$ is positive and $\|\AA\|^{2} \leq \varepsilon H^{2}$ at $p$ with $\epsilon<\frac{1}{n(n-1)}$, then the second fundamental form is positive definite at $p$, and the principal curvatures satisfy

$$
(1-\sqrt{n(n-1) \epsilon}) \frac{H}{n} \leq \kappa_{i} \leq(1+\sqrt{n(n-1) \epsilon}) \frac{H}{n} .
$$

Proof. Fix $i \in\{1, \ldots, n\}$. Then we have

$$
\begin{aligned}
0 & \geq\|A\|^{2}-\left(\frac{1}{n}+\epsilon\right) H^{2} \\
& =\kappa_{i}^{2}+\sum_{j \neq i} \kappa_{j}^{2}-\left(\frac{1}{n}+\epsilon\right) H^{2} \\
& \geq \kappa_{i}^{2}+\frac{1}{n-1}\left(\sum_{j \neq i} \kappa_{j}\right)^{2}-\left(\frac{1}{n}+\epsilon\right) H^{2} \\
& =\kappa_{i}^{2}+\frac{1}{n-1}\left(H-\kappa_{i}\right)^{2}-\left(\frac{1}{n}+\epsilon\right) H^{2} \\
& =\frac{n}{n-1} \kappa_{i}^{2}-\frac{2}{n-1} \kappa_{i} H+\left(\frac{1}{n(n-1)}-\epsilon\right) H^{2} .
\end{aligned}
$$

It follows that $z=n \kappa_{i} / H$ lies between the roots of the equation $z^{2}-2 z+(1-$ $n(n-1) \epsilon)=0$.

Define $C=\kappa_{1}^{3}+\cdots+\kappa_{n}^{3}$. The following estimate generalizes one from $\underline{\mathrm{H} 2}$.

Lemma 2.3. If $H>0$ and $\|\AA\|^{2}=\epsilon H^{2}$ at $p$ for some $\epsilon \in\left(0, \frac{1}{n(n-1)}\right)$, then

$$
n C-(1+n \epsilon) H\|A\|^{2} \geq \epsilon(1+n \epsilon)(1-\sqrt{n(n-1) \epsilon}) H^{3} .
$$

Proof. Let $Q=\|\AA\|^{2}-\epsilon H^{2}=\|A\|^{2}-\left(\frac{1}{n}+\epsilon\right) H^{2}$. Then $\dot{Q}^{i}:=\frac{\partial Q}{\partial \kappa_{i}}=2\left(\kappa_{i}-\left(\frac{1}{n}+\epsilon\right) H\right)$, and $\frac{n}{2} \sum_{i} \dot{Q}^{i} \kappa_{i}^{2}=n C-(1+n \epsilon) H\|A\|^{2}$. Noting that by Euler's relation $\sum_{i} \dot{Q}^{i} \kappa_{i}=$ 
$2 Q=0$, we write

$$
\begin{aligned}
& n C-(1+n \epsilon) H\|A\|^{2}=\frac{n}{2} \sum_{i} \dot{Q}^{i} \kappa_{i}^{2} \\
&=\frac{n}{2} \sum_{i} \dot{Q}^{i} \kappa_{i}\left(\left(\kappa_{i}-\left(\frac{1}{n}+\epsilon\right) H\right)+\left(\frac{1}{n}+\epsilon\right) H\right) \\
&=n \sum_{i} \kappa_{i}\left(\kappa_{i}-\left(\frac{1}{n}+\epsilon\right) H\right)^{2}+\frac{n}{2}\left(\frac{1}{n}+\epsilon\right) H \sum_{i} \dot{Q}^{i} \kappa_{i} \\
& \geq(1-\sqrt{n(n-1) \epsilon}) H \sum_{i}\left(\kappa_{i}-\left(\frac{1}{n}+\epsilon\right) H\right)^{2} \\
&=(1-\sqrt{n(n-1) \epsilon}) H\left(\|A\|^{2}-2\left(\frac{1}{n}+\epsilon\right) H^{2}\right. \\
&\left.\quad+n\left(\frac{1}{n}+\epsilon\right)^{2} H^{2}\right) \\
&=(1-\sqrt{n(n-1) \epsilon}) \epsilon(1+n \epsilon) H^{3},
\end{aligned}
$$

where we used the estimate of Lemma 2.2 to obtain the inequality.

\section{Geometric estimates}

In [A1, Theorem 5.1] it was proved that a pointwise bound on the ratio of principal curvatures of a convex hypersurface implies a bound on the ratio of maximum to minimum width. Our main result is a strengthening of that estimate in the case where the ratios of principal curvatures approach one at points where the maximum principal curvature is large. We denote by $r_{-}(M)$ the inradius of a convex hypersurface $M$ (the radius of the largest ball enclosed by $M$ ) and by $r_{+}(M)$ the circumradius (the radius of the smallest closed ball which contains $M$ ).

Theorem 3.1. Let $C_{1}(\varepsilon) \geq 0$ for each $\varepsilon>0$. Then for any $\rho>0$ there exists $R(\rho)>0$ such that every smooth convex compact hypersurface $M$ satisfying $\kappa_{n} \leq$ $(1+\varepsilon) \kappa_{1}+C_{1}(\varepsilon)$ for every $\varepsilon>0$ and $r_{+}(M) \leq R(\rho)$ satisfies $r_{+}(M) \leq(1+$ $\rho) r_{-}(M)$.

Proof. Define the (normalized) $k$-dimensional mean cross-sectional volume $V_{k}(M)$ of $M$ by

$$
V_{k}(M)=\frac{1}{\left|S^{n}\right|} \int_{M} E_{n-k} d \mu(g)
$$

if $0 \leq k \leq n$, and by

$$
V_{k}(M)=\frac{1}{\left|S^{n}\right|} \int_{M} s E_{n+1-k} d \mu(g)
$$

for $1 \leq k \leq n+1$, where $s=\langle X, \nu\rangle$ is the support function.

The ratio of the circumradius to the inradius of a convex body can be estimated in terms of the mean cross-sectional volumes. We will only need the following rather crude statement. 
Lemma 3.2. For any $\varepsilon>0$ there exists $\delta(\varepsilon, n)>0$ such that any convex body satisfying

$$
\frac{V_{1}^{n+1}}{V_{n+1}} \leq 1+\delta
$$

has

$$
\frac{r_{+}(M)}{r_{-}(M)} \leq 1+\varepsilon
$$

Proof. Since both $r_{+} / r_{-}$and $V_{1}^{n+1} / V_{n+1}$ are scaling invariant, it suffices to assume $V_{n+1}=1$. The Diskant inequalities [S1, Theorem 6.2.3] give estimates for the inradius and the circumradius:

$$
r_{-}(M) \geq V_{n}^{\frac{1}{n}}-\left(V_{n}^{\frac{n+1}{n}}-1\right)^{\frac{1}{n+1}} \quad \text { and } \quad r_{+}(M) \leq \frac{1}{V_{1}^{\frac{1}{n}}-\left(V_{1}^{\frac{n+1}{n}}-1\right)^{\frac{1}{n+1}}} .
$$

The function $f(x)=x-\left(x^{n+1}-1\right)^{\frac{1}{n+1}}$ is decreasing in $x$, so the inequality $V_{n} \leq V_{1}^{n}$ implies

This gives

$$
r_{-}(M) \geq V_{1}-\left(V_{1}^{n+1}-1\right)^{\frac{1}{n+1}} .
$$

$$
\frac{r_{-}(M)}{r_{+}(M)} \geq\left(V_{1}-\left(V_{1}^{n+1}-1\right)^{\frac{1}{n+1}}\right)\left(V_{1}^{\frac{1}{n}}-\left(V_{1}^{\frac{n+1}{n}}-1\right)^{\frac{1}{n+1}}\right) .
$$

The lemma follows since the right-hand side approaches 1 as $V_{1}$ approaches 1 .

In view of the lemma, it suffices to show that the isoperimetric ratio $V_{1}^{n+1} / V_{n+1}$ can be made close to 1 by requiring the inradius to be small. To do this we first observe that the elementary symmetric functions can be compared. For $1 \leq k<$ $\ell \leq n$ we can write (since $\kappa_{1}^{\ell} \leq E_{\ell}$ for any $\ell$ )

$$
\begin{aligned}
E_{k} \leq \kappa_{n}^{k} & \leq\left((1+\varepsilon) \kappa_{1}+C_{1}(\varepsilon)\right)^{k} \\
& \leq(1+\varepsilon)^{2 k}\left(\frac{1}{1+\varepsilon} E_{\ell}^{1 / \ell}+\left(1-\frac{1}{1+\varepsilon}\right) \frac{C_{1}(\varepsilon)}{\varepsilon}\right)^{k} \\
& \leq(1+\varepsilon)^{2 k-1} E_{\ell}^{k / \ell}+\frac{(1+\varepsilon)^{2 k-1}}{\varepsilon^{k-1}} C_{1}(\varepsilon)^{k},
\end{aligned}
$$

where we used the convexity of $x^{k}$ in the last step. Replacing $\varepsilon$ by $(1+\varepsilon)^{1 /(2 k-1)}-1$, we find

$$
E_{k} \leq(1+\varepsilon) E_{\ell}^{k / \ell}+C_{k}(\varepsilon),
$$

where $C_{k}(\varepsilon)=\frac{1+\varepsilon}{\left((1+\varepsilon)^{1 /(2 k-1)}-1\right)^{k-1}} C_{1}\left((1+\varepsilon)^{1 /(2 k-1)}-1\right)^{k}$. Integrating (3.1) with $k=n-1, \ell=n$ and dividing by $\left|S^{n}\right|$ gives:

$$
\begin{aligned}
V_{1}=\frac{1}{\left|S^{n}\right|} \int_{M} E_{n-1} d \mu(g) & \leq \frac{(1+\varepsilon)}{\left|S^{n}\right|} \int_{M} E_{n}^{1-1 / n} d \mu(g)+C_{n-1}(\varepsilon) V_{n} \\
& \leq(1+\varepsilon)\left(\frac{1}{\left|S^{n}\right|} \int_{M} E_{n} d \mu\right)^{1-1 / n} V_{n}^{1 / n}+C_{n-1}(\varepsilon) V_{n} \\
& =(1+\varepsilon) V_{n}^{1 / n}+C_{n-1}(\varepsilon) V_{n} \\
& \leq(1+\varepsilon) V_{n}^{1 / n}\left(1+\frac{C_{n-1}(\varepsilon)}{1+\varepsilon} r_{+}^{n-1}\right) .
\end{aligned}
$$


Next take (3.1) for $k=1$ and $\ell=n$, multiply by $s /\left|S^{n}\right|$, and integrate over $M$, yielding

$$
\begin{aligned}
V_{n}=\frac{1}{\left|S^{n}\right|} \int_{M} E_{1} s d \mu & \leq \frac{1+\varepsilon}{\left|S^{n}\right|} \int_{M} E_{n}^{1 / n} s d \mu(g)+C_{1}(\varepsilon) V_{n+1} \\
& \leq(1+\varepsilon)\left(\frac{1}{\left|S^{n}\right|} \int_{M} E_{n} s d \mu(g)\right)^{1 / n} V_{n+1}^{\frac{n-1}{n}}+C_{1}(\varepsilon) V_{n+1} \\
& =(1+\varepsilon) V_{1}^{\frac{1}{n}} V_{n+1}^{\frac{n-1}{n}}+C_{1}(\varepsilon) V_{n+1} \\
& \leq(1+\varepsilon) V_{1}^{\frac{1}{n}} V_{n+1}^{\frac{n-1}{n}}\left(1+\frac{C_{1}(\varepsilon)}{1+\varepsilon} r_{+}\right)
\end{aligned}
$$

where we used the Minkowski inequality $V_{n+1} \leq V_{1}^{n+1}$ in the last line [S1, Theorem 6.2.1]. From (3.2) and (3.3) we have

$$
V_{1}^{n+1} \leq(1+\varepsilon)^{\frac{n^{2}+n}{n-1}} V_{n+1}\left(1+\frac{C_{n-1}(\varepsilon)}{1+\varepsilon} r_{+}^{n-1}\right)^{\frac{n^{2}}{n-1}}\left(1+\frac{C_{1}(\varepsilon)}{1+\varepsilon} r_{+}\right)^{\frac{n}{n-1}},
$$

for any $\varepsilon>0$. Given $\rho>0$, choose $\varepsilon>0$ such that $(1+\varepsilon)^{\left(n^{2}+n\right) /(n-1)}<\sqrt{1+\rho}$, and then choose $R(\rho)$ such that $\left(1+\frac{C_{n-1}(\varepsilon)}{1+\varepsilon} R(\rho)^{n-1}\right)^{n}\left(1+\frac{C_{1}(\varepsilon)}{1+\varepsilon} R(\rho)\right) \leq(1+\rho)^{\frac{n-1}{2 n}}$. The theorem follows.

Remark. $\quad$ 1. A similar but simpler argument using the Diskant inequalities proves a result similar to [A1, Theorem 5.1]. The precise statement is rather messier than in [A1, but it implies the ratio of $r_{+}(M)$ to $r_{-}(M)$ approaches one if the pointwise ratio of principal curvatures approaches one. The proof in A1 gives this only for ratios of widths.

2. An anisotropic analogue also holds: If $W$ is a smooth, uniformly convex Wulff shape enclosing the origin, and if $M$ is a smooth convex hypersurface, then the relative normal $\nu_{W}: M \rightarrow W$ takes a point in $M$ to the unique point in $W$ with the same outward normal vector. The derivative of $\nu_{W}$ is the relative Weingarten map, the eigenvalues $\kappa_{1}, \ldots, \kappa_{n}$ of which are the relative principal curvatures. The relative support function $s_{W}$ is defined by $X=s_{W} \nu_{W}+V$, where $V$ is tangent to $M$, and is given by $s_{W}=\frac{\langle X, \nu\rangle}{\left\langle\nu_{W}, \nu\right\rangle}$. The relative $k$-dimensional volumes $V_{k}(M, W)$ are then defined by exactly the same formulae as $V_{k}(M)$ above, where $E_{k}$ is the elementary symmetric function of the relative principal curvatures. The relative inradius $r_{-}(M)$ is the supremum of all $r$ such that a translate of $r W$ can be enclosed by $M$, and the relative cirumradius $r_{+}(M)$ is the infimum of all $r$ such that a translate of $r W$ encloses $M$. Then Theorem 3.1 holds as stated (we remark that the Diskant inequality applies unchanged in this situation).

\section{The FLOW EQUATIONS}

Given a smooth, compact, uniformly convex initial hypersurface $M_{0}^{n}=X_{0}(M)$ with $n \geq 2$, we consider a family of embeddings $X: M \times[0, T) \rightarrow \mathbb{R}^{n+1}$ satisfying the evolution equation

$$
\frac{\partial X}{\partial t}(x, t)=-F(A(x, t)) \nu(x, t)
$$


where $\nu$ denotes the outer unit normal to the evolving hypersurface $M_{t}=X(M, t)$ $=X_{t}(M)$, and $F$ is an $O(n)$-invariant function of $A$ with the following properties:

Conditions 4.1. $\quad$ (i) $F=f \circ \kappa$, where $\kappa$ is the map which takes a selfadjoint operator to its ordered eigenvalues, and $f$ is a smooth, symmetric function defined on an open symmetric cone $\Gamma \subseteq \Gamma_{+}$containing $\{(c, \ldots, c): c>0\}$, where $\Gamma_{+}=\left\{\kappa: \kappa_{i}>0, i=1, \ldots, n\right\}$.

(ii) $f$ is strictly monotone: $\frac{\partial f}{\partial \kappa_{i}}>0$ for each $i=1, \ldots, n$, at each point of $\Gamma$.

(iii) $f$ is homogeneous of degree $\alpha>1: f(k \kappa)=k^{\alpha} f(\kappa)$ for any $k>0$.

(iv) $f$ is normalized to have $f(1, \ldots, 1)=n^{\alpha}$.

Remark. $\quad 1$. Since $f$ is a smooth symmetric function by Condition 4.1, it is also a smooth function of the elementary symmetric functions of the principal curvatures [G], so $F$ is a smooth function of the components of $A$ and $g$.

2. It follows from homogeneity (Condition 4.1), strict monotonicity (Condition 4.1) and the Euler relation $\alpha F=\sum_{i} \kappa_{i} \frac{\partial f}{\partial \kappa_{i}}$ that $F$ is strictly positive on the interior of $\Gamma \cap \Gamma_{+}$. The normalization in Condition 4.1 is for convenience and can be achieved by scaling the time parameter in equation (4.1). The particular choice is made to agree with powers of the mean curvature.

3. There are many examples of natural flows satisfying these conditions which are not covered by previous results, for example, powers of ratios of elementary symmetric functions of curvature, and sums and products of such, and the power means $f=\left(\sum_{i=1}^{n} \kappa_{i}^{r}\right)^{\alpha / r}$ for $r \neq 0,1$; however, the smoothness assumptions rule out some examples which are strictly parabolic and concave, such as $f=\left(H+\kappa_{\min }\right)^{\alpha}$.

4. Notably absent in our assumptions is any concavity or convexity of $f$. This is usually indispensible as a condition of this kind is normally required to derive second derivative Hölder estimates for fully nonlinear parabolic equations. However the situation in the present paper is sufficiently restricted that we can apply a result of Cordes-Nirenberg type which requires no concavity. The required arguments are provided in Section 7.

Throughout this paper we sum over repeated indices from 1 to $n$ unless otherwise indicated. In computations on the hypersurface $M_{t}$, raised indices indicate contraction with the metric.

We denote by $\dot{F}^{k l}$ the derivatives of $F$ with respect to the components of its argument:

$$
\left.\frac{d}{d s} F(A+s B)\right|_{s=0}=\left.\dot{F}^{k l}\right|_{A} B_{k l} .
$$

Similarly for the second partial derivatives of $F$ we write

$$
\left.\frac{d^{2}}{d s^{2}} F(A+s B)\right|_{s=0}=\left.\ddot{F}^{k l, r s}\right|_{A} B_{k l} B_{r s} .
$$

Here and throughout the paper we sum over repeated indices. If we do not indicate explicitly where derivatives of $F$ and of $f$ are evaluated then they are evaluated at $A$ and $\kappa(A)$ respectively. We will use similar notation and conventions for derivatives of other functions of matrices. 
Our later choices and estimates will be determined by two constants $\delta_{0}$ and $\mu$ depending on $F$ and $\Gamma$. Since $\Gamma$ is open, there exists $\delta_{0} \in\left(0, \frac{1}{n(n-1)}\right)$ such that $\Gamma_{0}=\left\{\kappa(A):\|\AA\|^{2} \leq \delta_{0} H^{2}\right\} \subset \Gamma$. Since $F$ is smooth and the set $\{A: \kappa(A) \in$ $\left.\Gamma_{0}, \operatorname{tr}(A)=1\right\}$ is compact, there exists $\mu \geq 0$ such that for any $A$ with $H=\operatorname{tr} A=1$ and $\kappa(A) \in \Gamma_{0}$, we have $\left|\ddot{F}^{k l, r s}\right|_{A} B_{k l} B_{r s} \mid \leq \mu\|B\|^{2}$. It follows that for arbitrary $A$ with $\kappa(A) \in \Gamma_{0}$ and arbitrary symmetric 2-tensors $B$ and $C$,

$$
\left|\ddot{F}^{k l, r s}\right|_{A} B_{k l} C_{r s} \mid \leq \mu H^{\alpha-2}\|B\|\|C\| .
$$

We can deduce from this good control on the first derivatives of $F$. By the normalization in Condition 4.114.1, we have $\left.\dot{F}\right|_{\frac{H}{n} I}=\alpha H^{\alpha-1} I$. Integrating and using the estimate (4.2) gives

$$
\begin{aligned}
|\dot{F}|_{A}(B)-\alpha H^{\alpha-1} \operatorname{tr}(B) \mid & \leq \int_{0}^{1}\left|\frac{d}{d s} \dot{F}\right|_{\frac{H}{n} I+s \AA}(B) \mid d s \\
& =\int_{0}^{1}|\ddot{F}|_{\frac{H}{n} I+s \AA}(B, \AA) \mid d s \\
& \leq \mu H^{\alpha-2}\|\AA\|\|B\| .
\end{aligned}
$$

Maximizing over rank one operators $B$ gives the inequalities

$$
\left(\alpha H^{\alpha-1}-\mu H^{\alpha-2}\|\AA\|\right) I \leq \dot{F} \leq\left(\alpha H^{\alpha-1}+\mu H^{\alpha-2}\|\AA\|\right) I .
$$

Equation (4.2) also gives good control on the values of $F$ itself. Using the degree two

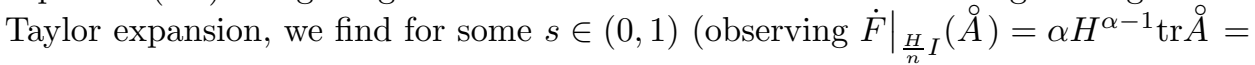
$0)$ :

$$
\left|F(A)-H^{\alpha}\right|=|F(A)-F|_{\frac{H}{n} I}-\left.\left.\dot{F}\right|_{\frac{H}{n} I}(\AA)\left|=\frac{1}{2}\right| \ddot{F}\right|_{\frac{H}{n} I+s \AA}(\AA, \AA) \mid \leq \frac{\mu}{2} H^{\alpha-2}\|\AA\|^{2} .
$$

Our main result regarding the flows is the following:

Theorem 4.2. Let $F$ satisfy Conditions 4.1, and define $\delta_{0}$ and $\mu$ as above. Then there exists $\delta>0$ depending only on $n, \alpha$ and $\mu$ such that for any smooth, uniformly locally convex embedding $X_{0}: M^{n} \rightarrow \mathbb{R}^{n+1}$ of a compact manifold $M$ satisfying $\|\AA\|^{2}<\min \left\{\delta, \delta_{0}\right\} H^{2}$, there exists a unique solution $X: M \times[0, T) \rightarrow \mathbb{R}^{n+1}$ of equation (4.1) with initial data $X_{0}$, and a point $p \in \mathbb{R}^{n+1}$ such that $X(M, t) \rightarrow p$ as $t \rightarrow T$, and $\tilde{X}_{t}=\frac{X(., t)-p}{((1+\alpha)(T-t))^{1 /(1+\alpha)}}$ converges in $C^{\infty}$ to an embedding $X_{\infty}: M \rightarrow$ $\mathbb{R}^{n+1}$ with $X_{\infty}(M)=S_{1}^{n}(0)$.

Short time existence of a solution to equation (4.1) for smooth, uniformly convex initial data is well known (see [A1, for example). The following evolution equations are derived as in $\mathrm{A} 1$.

Lemma 4.3. Under the flow (4.1), the following evolution equations hold:

(i) $\frac{\partial}{\partial t}\langle X, \nu\rangle=\mathcal{L}\langle X, \nu\rangle+\langle X, \nu\rangle \dot{F}^{k l} h_{k m} h^{m}{ }_{l}-(1+\alpha) F$;

(ii) $\frac{\partial}{\partial t} h_{j}^{i}=\mathcal{L} h^{i}{ }_{j}+\ddot{F}^{k l, r s} \nabla^{i} h_{k l} \nabla_{j} h_{r s}+\dot{F}^{k l} h_{k m} h^{m}{ }_{l} h^{i}{ }_{j}+(1-\alpha) F h^{i m} h_{m j}$, and 
(iii) for any smooth symmetric function $G(\mathcal{W})=g(\kappa(\mathcal{W}))$,

$$
\begin{aligned}
\frac{\partial}{\partial t} G= & \mathcal{L} G+\left[\dot{G}^{i j} \ddot{F}^{k l, r s}-\dot{F}^{i j} \ddot{G}^{k l, r s}\right] \nabla_{i} h_{k l} \nabla_{j} h_{r s} \\
& +\dot{F}^{k l} h_{k m} h^{m}{ }_{l} \dot{G}^{i j} h_{i j}+(1-\alpha) F \dot{G}^{i j} h_{i m} h^{m}{ }_{j},
\end{aligned}
$$

where $\mathcal{L} G=\dot{F}^{i j} \nabla_{i} \nabla_{j} G$. In particular

(iv) $\frac{\partial}{\partial t} F=\mathcal{L} F+\dot{F}^{k l} h_{k m} h^{m}{ }_{l} F$, and

(v) $\frac{\partial}{\partial t} H=\mathcal{L} H+\ddot{F}^{k l, r s} \nabla^{i} h_{k l} \nabla_{i} h_{r s}+\dot{F}^{k l} h_{k m} h^{m}{ }_{l} H+(1-\alpha) F\|A\|^{2}$.

\section{Preserving PINChING}

In this section we prove the following for solutions of equation (4.1):

Theorem 5.1. There exists $\delta_{1} \in\left(0, \frac{1}{n(n-1)}\right)$ depending on $n, \mu$ and $\alpha$ such that if $0<\sigma \leq \min \left\{\delta_{0}, \delta_{1}\right\}$ and $\|\AA\|^{2}<\sigma H^{2}$ at every point at $t=0$, then this remains true for $t>0$.

Proof. Using Lemma 4.3 we compute the evolution equation for $Z_{\sigma}=\|\AA\|^{2}-\sigma H^{2}$ :

$$
\begin{aligned}
\frac{\partial Z_{\sigma}}{\partial t}= & \mathcal{L} Z_{\sigma}+2\left(h^{i j}-\left(\frac{1}{n}+\sigma\right) H g^{i j}\right) \ddot{F}^{k l, r s} \nabla_{i} h_{k l} \nabla_{j} h_{r s} \\
& -2 \dot{F}^{i j}\left(\nabla_{i} h_{k l} \nabla_{j} h^{k l}-\frac{1}{n} \nabla_{i} H \nabla_{j} H\right) \\
& +2 \sigma \dot{F}^{i j} \nabla_{i} H \nabla_{j} H+2 \dot{F}^{i j} h_{i m} h_{j}^{m} Z_{\sigma} \\
& +\frac{2}{n}(1-\alpha) F\left(n C-(1+n \sigma) H\|A\|^{2}\right) .
\end{aligned}
$$

We argue that $Z_{\sigma}$ remains negative if it is initially so. Otherwise there exists a first point and time $\left(x_{0}, t_{0}\right)$ with $Z_{\sigma}=0$, where $\mathcal{L} Z_{\sigma} \leq 0$ and $\partial_{t} Z_{\sigma} \geq 0$. The second to last term in (5.1) is zero at $\left(x_{0}, t_{0}\right)$ since $Z_{\sigma}=0$, and the last is negative by Lemma 2.3. It suffices to show that the terms involving $\nabla h$ are nonpositive, since then the right-hand side is negative while the left is nonnegative.

We estimate the $\ddot{F}$ term using equation (4.2):

$$
\begin{aligned}
2\left|\left[h^{i j}-\left(\frac{1}{n}+\sigma\right) H g^{i j}\right] \ddot{F}^{k l, r s} \nabla_{i} h_{k l} \nabla_{j} h_{r s}\right| & \leq 2\left\|h^{i j}-\left(\frac{1}{n}+\sigma\right) H g^{i j}\right\|\|\ddot{F}\|\|\nabla A\|^{2} \\
& \leq 2 \sqrt{\sigma(1+n \sigma)} H\|\ddot{F}\|\|\nabla A\|^{2} \\
& \leq 2 \sqrt{\sigma(1+n \sigma)} H^{\alpha-1} \mu\|\nabla A\|^{2} .
\end{aligned}
$$

The next term is the good negative term which we estimate as follows:

$$
\begin{aligned}
\dot{F}^{i j}\left[\nabla_{i} h_{k l} \nabla_{j} h^{k l}-\frac{1}{n} \nabla_{i} H \nabla_{j} H\right] & =\dot{F}^{i j}\left(\nabla_{i} h_{k l}-\frac{1}{n} g_{k l} \nabla_{i} H\right)\left(\nabla_{j} h^{k l}-\frac{1}{n} g^{k l} \nabla_{j} H\right) \\
& \geq\left(\alpha H^{\alpha-1}-\mu H^{\alpha-2}\|\AA\|\right)\|\nabla \AA\|^{2} \\
& \geq \frac{2(n-1)}{3 n} H^{\alpha-1}(\alpha-\mu \sqrt{\sigma})\|\nabla A\|^{2},
\end{aligned}
$$


where we used equation (4.3) and Lemma 2.1. The same estimates also control the remaining term:

$$
\begin{aligned}
\left|2 \sigma \dot{F}^{i j} \nabla_{i} H \nabla_{j} H\right| & \leq 2 \sigma(\alpha+\mu \sqrt{\sigma}) H^{\alpha-1}\|\nabla H\|^{2} \\
& \leq \frac{2(n+2)}{3} \sigma(\alpha+\mu \sqrt{\sigma}) H^{\alpha-1}\|\nabla A\|^{2} .
\end{aligned}
$$

Putting these estimates together, the gradient terms at $\left(p_{0}, t_{0}\right)$ in (5.1) are no greater than

$$
\left(-\frac{4(n-1)}{3 n}(\alpha-\mu \sqrt{\sigma})+2 \sqrt{\sigma(1+n \sigma)} \mu+\frac{2(n+2)}{3} \sigma(\alpha+\mu \sqrt{\sigma})\right) H^{\alpha-1}\|\nabla A\|^{2} .
$$

The bracket is clearly nonpositive on $0 \leq \sigma \leq \delta_{1}$ for some $\delta_{1}>0$ depending on $n$, $\mu$ and $\alpha$.

Remark 5.2. By Lemma 4.3, the minimum of $F$ does not decrease in $t$. Since $\left(n \kappa_{\max }\right)^{\alpha}=f\left(\kappa_{\max }, \ldots, \kappa_{\max }\right) \geq f\left(\kappa_{1}, \ldots, \kappa_{n}\right), \kappa_{\max }$ remains strictly positive and $H>0$ by Lemma 2.2 .

Remark 5.3. We used the second derivative bound $\mu$, but no concavity of $f$. Onesided second derivative bounds might suffice, but mere parabolicity does not (unless $n=2$ as shown in [A4]); for example, no pinching cones exist if $F=\left(H+c \kappa_{2}\right)^{\alpha}$ with $c>3$ for $n=3$.

\section{Curvature Bound}

Next we prove upper bounds on the speed, by using an argument of Tso [T] to show that the speed remains bounded if the inradius stays positive. Suppose $r_{-}\left(M_{t}\right) \geq r_{0}$ for $0 \leq t \leq t_{0}$. Take the origin to be the centre of the insphere of $M_{t_{0}}$, so that $M_{t}$ encloses $B_{r_{0}}(0)$ for $0 \leq t \leq t_{0}$. Let $Q=\frac{F}{2\langle X, \nu\rangle-r_{0}}$. By Theorem 5.1 and Lemma 2.2 $\kappa_{\min } \geq \frac{1-\sqrt{n(n-1) \sigma}}{1+\sqrt{n(n-1) \sigma}} \kappa_{\max }$ with $\sigma<\min \left\{\delta_{0}, \delta_{1}\right\}$, so

$$
\dot{F}^{k l} h_{k}{ }^{p} h_{p l} \geq \alpha \kappa_{\min } F \geq \frac{\alpha(1-\sqrt{n(n-1) \sigma})}{n(1+\sqrt{n(n-1) \sigma})} F^{1+1 / \alpha},
$$

where we observed $F=f\left(\kappa_{1}, \ldots, \kappa_{n}\right) \leq f\left(\kappa_{\max }, \ldots, \kappa_{\max }\right)=\left(n \kappa_{\max }\right)^{\alpha}$. Using this we compute

$$
\begin{aligned}
\frac{\partial Q}{\partial t} & =\mathcal{L} Q+4 \dot{F}^{k l} \frac{\nabla_{k}\langle X, \nu\rangle}{2\langle X, \nu\rangle-r_{0}} \nabla_{l} Q-\frac{F}{\left(2\langle X, \nu\rangle-r_{0}\right)^{2}}\left(r_{0} \dot{F}^{k l} h_{k}{ }^{p} h_{p l}-2(1+\alpha) F\right) \\
& \leq \mathcal{L} Q+4 \dot{F}^{k l} \frac{\nabla_{k}\langle X, \nu\rangle}{2\langle X, \nu\rangle-r_{0}} \nabla_{l} Q+2(1+\alpha) Q^{2}-r_{0}^{1+1 / \alpha} \tilde{C} Q^{2+1 / \alpha},
\end{aligned}
$$

where $\tilde{C}=\frac{\alpha(1-\sqrt{n(n-1) \sigma})}{n(1+\sqrt{n(n-1) \sigma}}$, and we used $2\langle X, \nu\rangle-r_{0} \geq r_{0}$. The maximum principle then implies

$$
Q \leq \max \left\{\left(\frac{2(1+\alpha)}{\tilde{C}}\right)^{\alpha} r_{0}^{-(1+\alpha)},\left(\frac{(1+\alpha) \tilde{C}}{2 \alpha}\right)^{-\alpha /(1+\alpha)} r_{0}^{-1} t^{-\alpha /(1+\alpha)}\right\}
$$

It follows that $F$ is bounded, and hence so is $\kappa_{\max }$. We have proved the following. 
Proposition 6.1. Let $X: M \times\left[0, t_{0}\right] \rightarrow \mathbb{R}^{n+1}$ be a solution of (4.1) with $\|\AA\|^{2}<$ $\min \left\{\delta_{0}, \delta_{1}\right\} H^{2}$ at $t=0$. Then there exists $C_{+}$depending only on $n, \alpha, \mu, r_{+}\left(M_{0}\right)$ and $r_{-}\left(M_{t_{0}}\right)$ such that $\kappa_{\max }(x, t) \leq C_{+}\left(1+t^{-1 /(1+\alpha)}\right)$ for all $(x, t) \in M \times\left(0, t_{0}\right]$.

\section{REgULARITY RESULTS FOR PARABOLIC EQUATIONS}

Here we summarise the ingredients needed for our regularity results. Denote by $Q_{r}$ the space-time cylinder $B_{r}(0) \times\left(-r^{2}, 0\right]$. We need the result on Hölder continuity of solutions of linear uniformly parabolic equations by Krylov and Safonov [KS] (see also [L, Corollary 7.26]):

Theorem 7.1. For any $0<\lambda \leq \Lambda<\infty$ and $B \geq 0$, there exist $\gamma \in(0,1)$ and $K$ such that for any smooth solution $u: Q_{1} \rightarrow \mathbb{R}$ of the equation

$$
\frac{\partial u}{\partial t}=a^{i j} \frac{\partial^{2} u}{\partial x^{i} \partial x^{j}}+b^{i} \frac{\partial u}{\partial x^{i}}+f
$$

with $\lambda\|\xi\|^{2} \leq a^{i j} \xi_{i} \xi_{j} \leq \Lambda\|\xi\|^{2}$ for all $\xi$ and $\left|b^{i}\right| \leq B$, the following holds for $0<r<1$ :

$$
\underset{Q_{r}}{\operatorname{Osc}} u \leq K r^{\gamma}\left(\underset{Q_{1}}{\operatorname{Osc}} u+\|f\|_{\infty}\right)
$$

Schauder estimates give more regularity if the coefficients are regular (see $[\mathrm{L}$, Theorem 4.9]):

Theorem 7.2. Let $0<\lambda \leq \Lambda<\infty, A, B, C \geq 0$, and $\beta \in(0,1)$. Then there exists $K$ depending only on $n, \lambda, \Lambda, A, B, C$ and $\beta$ such that for any smooth solution $u: Q_{1} \rightarrow \mathbb{R}$ of the equation

$$
\frac{\partial u}{\partial t}=a^{i j} \frac{\partial^{2} u}{\partial x^{i} \partial x^{j}}+b^{i} \frac{\partial u}{\partial x^{i}}+c u+f,
$$

where $\lambda\|\xi\|^{2} \leq a^{i j} \xi_{i} \xi_{j} \leq \Lambda\|\xi\|^{2}$ for all $\xi,\left|a^{i j}(x, t)-a^{i j}(y, s)\right| \leq A \max \left\{|x-y|^{\beta}\right.$, $\left.|t-s|^{\beta / 2}\right\},\left|b^{i}(x, t)\right| \leq B,\left|b^{i}(x, t)-b^{i}(y, s)\right| \leq B \max \left\{|x-y|^{\beta},|t-s|^{\beta / 2}\right\},|c(x, t)| \leq$ $C$ and $|c(x, t)-c(y, s)| \leq C \max \left\{|x-y|^{\beta},|t-s|^{\beta / 2}\right\}$ for all $(x, t)$ and $(y, s)$ in $B_{1}(0) \times[-1,0]$, the following estimate holds:

$$
\begin{aligned}
&\|D u\|_{L^{\infty}\left(Q_{1 / 2}\right)}+\left\|D^{2} u\right\|_{L^{\infty}\left(Q_{1 / 2}\right)}+\left\|\frac{\partial u}{\partial t}\right\|_{L^{\infty}\left(Q_{1 / 2}\right)} \\
&+\sup _{(x, t) \neq(y, s) \text { in } Q_{1 / 2}} \frac{\left|\frac{\partial u}{\partial t}(x, s)-\frac{\partial u}{\partial t}(y, s)\right|}{\max \left\{|x-y|^{\beta},|t-s|^{\beta / 2}\right\}} \\
&+\sup _{(x, t) \neq(y, s) \text { in } Q_{1 / 2}} \frac{\left|D^{2} u(x, t)-D^{2} u(y, s)\right|}{\max \left\{|x-y|^{\beta},|t-s|^{\beta / 2}\right\}} \\
&+\sup _{(x, t),(x, s) \in Q_{1 / 2}, t \neq s} \frac{|D u(x, t)-D u(x, s)|}{|t-s|^{(1+\beta) / 2}} \\
& \leq K\left(\|u\|_{L^{\infty}\left(Q_{1}\right)}+\|f\|_{L^{\infty}\left(Q_{1}\right)}+\sup _{(x, t) \neq(y, s) \text { in } Q_{1}} \frac{|f(x, t)-f(y, s)|}{\max \left\{|x-y|^{\beta},|t-s|^{\beta / 2}\right\}}\right) .
\end{aligned}
$$

In order to apply Schauder estimates to deduce higher regularity for solutions of fully nonlinear parabolic equations, one must first prove that derivatives up to 
second order in space and first order in time are Hölder continuous. In most such cases it is necessary to use the results of Krylov [K] (or Evans [E] for the elliptic case) which require concave dependence on the second derivatives. However in the situation of this paper the geometric restrictions allow us to dispense with this requirement. Cordes $\mathrm{Co}$ and Nirenberg $\mathrm{N}$. proved that linear elliptic equations with coefficients close to the identity admit $C^{1, \alpha}$ estimates. We use a result of this kind from [L, Lemma 12.13], proved by adapting to the parabolic setting a method of Caffarelli $\mathrm{Ca}$.

Theorem 7.3. Let $0<\lambda \leq \Lambda<\infty, A>0$ and $B>0$. Then there exist constants $C>0, \gamma \in(0,1], K \geq 0$ and $\sigma>0$ such that for any $u: Q_{1} \rightarrow \mathbb{R}$ with continuous first time derivatives and second spatial derivatives, satisfying the equation

$$
\frac{\partial u}{\partial t}=a^{i j}(x, t) D_{i} D_{j} u+a(x, t)
$$

with $\lambda \delta^{i j} \leq a^{i j}(x, t) \leq \Lambda \delta^{i j},|D u| \leq B,|a(x, t)| \leq A$, and $\left|a^{i j}(x, t)-a^{i j}(y, s)\right| \leq \sigma$ for all $(x, t)$ and $(y, s)$ in $Q_{1}$, we have for all $0<r<1$,

$$
\underset{Q_{r}}{\operatorname{osc}} D u \leq C r^{\gamma}\left(\underset{Q_{1}}{\operatorname{osc} D u+K}\right) .
$$

\section{HÖLDER CONTINUITY OF SECOND DERIVATIVES}

In this section we use the regularity results provided in the previous section to prove Hölder continuity of the second fundamental form. The proof proceeds in several stages. First, we observe that upper and lower bounds on $F$ together with the pinching result of Theorem 5.1 imply bounds above and below on second derivatives, which in turn imply that the evolving hypersurfaces can be described locally as the graph of a function evolving by a uniformly parabolic equation. From this we deduce that the unit normal $\nu$ and the speed $F$ are Hölder continuous in space-time. It follows that on small space-time regions the evolving graphs have first spatial derivatives satisfying an equation to which Theorem 7.3 can be applied, yielding the following:

Theorem 8.1. Let $F$ satisfy Conditions 4.1. Then there exist $\delta_{2} \in\left(0, \delta_{1}\right]$ and $\gamma \in(0,1]$ depending only on $n, \alpha$ and $\mu$ such that for any smooth uniformly convex solution $X: M \times[0, \tau] \rightarrow \mathbb{R}^{n+1}$ of (4.1) with $\|\AA\|^{2}<\min \left\{\delta_{2}, \delta_{0}\right\} H^{2}$ everywhere,

$\left|h_{x, t}(v, v)-h_{y, s}(w, w)\right| \leq C\left(|X(x, t)-X(y, s)|^{\gamma}+|t-s|^{\gamma / 2}+\left|X_{*}(v)-X_{*}(w)\right|^{\gamma}\right)$

for all $(x, t),(y, s) \in M \times[\tau / 2, \tau]$ with $|X(x, t)-X(y, s)|+\sqrt{|t-s|}<d, v \in T_{x} M$, and $w \in T_{y} M$ (here $X_{*}$ is the derivative of $X$, so $X_{*}(v)$ is the vector in $\mathbb{R}^{n+1}$ corresponding to $v)$, where $C$ and $d$ depend on $\tau, r_{+}=r_{+}\left(M_{0}\right), r_{-}=r_{-}\left(M_{\tau}\right)$ and $\inf _{M_{0}} F$.

Proof. We first deduce a curvature bound:

Lemma 8.2. For $X$ as in Theorem 8.1 there exist $0<k_{-}<k_{+}<\infty$ depending on $\tau, n, \mu, \alpha, r_{ \pm}$and $\inf _{M_{0}} F$ such that $k_{-} \leq \kappa_{i}(x, t) \leq k_{+}$for all $x \in M, t \in[\tau / 8, \tau]$ and $i \in\{1, \ldots, n\}$.

Proof. The uniform convexity of $M_{0}$ implies that the speed $F$ has a positive lower bound at $t=0$, and the evolution equation for $F$ in Lemma 4.34.3 implies that 
this lower bound is maintained as long as the solution exists. By Proposition 6.1 we deduce an upper bound on $F$ on $M \times[\tau / 8, \tau]$, depending only on $r_{-}\left(M_{\tau}\right)$ and $r_{+}\left(M_{0}\right)$. Theorem 5.1 and Lemma 2.2 imply upper and lower bounds on the principal curvatures.

In order to apply the results of the previous section we write the evolving hypersurface locally as an evolving graph: Choose $x_{1} \in M$ and $t_{1} \in[\tau / 8, \tau]$. Since $\kappa_{i} \leq k_{+}, M_{t_{1}}$ encloses a sphere of radius $1 / k_{+}$which touches at $X\left(x_{1}, t_{1}\right)$. Choose the origin to be at the centre of this sphere. Then $M_{t}$ lies outside the sphere of radius $\left(k_{+}^{-(1+\alpha)}-(1+\alpha)\left(t-t_{1}\right)\right)^{\frac{1}{1+\alpha}}$ for $t_{1} \leq t<t_{1}+\frac{1}{(1+\alpha) k_{+}^{1+\alpha}}$. In particular $M_{t}$ lies outside the sphere of radius $2^{-\frac{1}{1+\alpha}} k_{+}^{-1}$ for $t \in\left[t_{1}, t_{1}+\frac{1}{2(1+\alpha)} k_{+}^{-(1+\alpha)}\right]$. Choose an orthonormal basis for $\mathbb{R}^{n+1}$ with $e_{n+1}=k_{+} X\left(x_{1}, t_{1}\right)$. By convexity, we have

$$
\begin{aligned}
M_{t} \cap\left\{X \cdot e_{n+1}>0\right\} \cap\left\{\mid X-\left(e_{n+1} \cdot X\right)\right. & \left.e_{n+1} \mid<2^{-\frac{1}{1+\alpha}} k_{+}^{-1}\right\} \\
& =\left\{(z,-u(z, t)):|z|<2^{-\frac{1}{1+\alpha}} k_{+}^{-1}\right\},
\end{aligned}
$$

where $u \in C^{\infty}\left(B_{2^{-\frac{1}{1+\alpha}} k_{+}^{-1}}(0) \times\left[t_{1}, t_{1}+\frac{1}{2(1+\alpha)} k_{+}^{-(1+\alpha)}\right]\right)$ is convex for each $t$, $0 \geq u(z, t) \geq-k_{+}^{-1}$ and $|D u(z, t)| \leq 2^{1+\frac{1}{1+\alpha}}$ for $|z| \leq 2^{-\frac{1}{1+\alpha}} k_{+}^{-1}$. We bound the second spatial derivatives $D^{2} u$ : The second fundamental form is $h_{i j}=\frac{D_{i} D_{j} u}{\sqrt{1+|D u|^{2}}}$, and the induced metric is $g_{i j}=\delta_{i j}+D_{i} u D_{j} u$. The upper and lower bounds on principal curvatures give $k_{-} g_{i j} \leq h_{i j} \leq k_{+} g_{i j}$, which becomes

$$
k_{-}\left(\delta_{i j}+D_{i} u D_{j} u\right) \sqrt{1+|D u|^{2}} \leq D_{i} D_{j} u \leq k_{+}\left(\delta_{i j}+D_{i} u D_{j} u\right) \sqrt{1+|D u|^{2}},
$$

so that by the gradient bounds we have

$$
k_{-} \delta_{i j} \leq D_{i} D_{j} u \leq k_{+}\left(1+2^{2+\frac{2}{1+\alpha}}\right)^{\frac{3}{2}} .
$$

The function $u$ evolves according to the scalar parabolic evolution equation

$$
\frac{\partial u}{\partial t}=\left(1+|D u|^{2}\right)^{\frac{1-\alpha}{2}} F\left(P^{T} \circ D^{2} u \circ P\right)=: \hat{F}\left(D^{2} u, D u\right),
$$

where $P_{i j}=\delta_{i j}-\frac{D_{i} u D_{j} u}{\sqrt{1+|D u|^{2}}\left(1+\sqrt{1+|D u|^{2}}\right)}$ is the square root of the inverse of the induced metric.

Our next step is to prove that the unit normal vector is Hölder-continuous (in space-time):

Lemma 8.3. For $X$ as in Theorem 8.1, there exists $C$ and $d$ depending only on $r_{+}\left(M_{0}\right), r_{-}\left(M_{\tau}\right)$ and $\inf _{M_{0}} F$ such that

$$
|\nu(x, t)-\nu(y, s)| \leq C(|X(x, t)-X(y, s)|+\sqrt{|t-s|})
$$

for all $(x, t)$ and $(y, s)$ in $M \times[\tau / 8, \tau]$ with $|X(x, t)-X(y, s)|+\sqrt{|t-s|}<d$. 
Proof. Differentiating equation (8.2) in a spatial direction gives the following evolution equation for $v=\frac{\partial u}{\partial z^{i}}$ for any $i$ :

$$
\frac{\partial v}{\partial t}=a^{k l} D_{k} D_{l} v+b^{k} D_{k} v
$$

where $a^{k l}=\left.\frac{\partial \hat{F}(r, p)}{\partial r_{k l}}\right|_{(r, p)=\left(D^{2} u, D u\right)}$ and $b^{k}=\left.\frac{\partial \hat{F}(r, p)}{\partial p_{k}}\right|_{(r, p)=\left(D^{2} u, D u\right)}$. Explicitly we have

$$
a^{k l}=\left.\sqrt{1+|D u|^{2}} \dot{F}^{p q}\right|_{P^{T}\left(D^{2} u\right) P} P^{p k} P^{q l},
$$

and $b^{k}$ is an expression involving $D u$ and $D^{2} u$, which may be bounded as follows:

$$
\left|b^{k}\right| \leq C\left(|D u|,\left|D^{2} u\right|\right)|D u| .
$$

In particular $\left|b^{k}\right| \leq B$, where $B$ depends only on $r_{+}\left(M_{0}\right), r_{-}\left(M_{\tau}\right)$ and $\inf _{M_{0}} F$. We note that by equations (8.4) and (4.3) and (4.4), there exists $\Lambda>0$ depending on $\mu, n, \alpha$ and $r_{ \pm}$such that $a^{i j} \xi_{i} \xi_{j} \leq \Lambda\|\xi\|^{2}$ for all $\xi$.

Equation 8.1provides Lipschitz dependence of $v$ in space. We use a barrier argument to deduce the required continuity in time: Since $v\left(0, t_{1}\right)=0$, and $|v(x, t)| \leq$ $2^{1+\frac{1}{1+\alpha}}$, and $|D v(x, t)| \leq K=k_{+}\left(1+2^{2+\frac{2}{1+\alpha}}\right)^{\frac{3}{2}}$ everywhere on $B_{2^{-\frac{1}{1+\alpha}}} k_{+}^{-1}(0) \times$ $\left[t_{1}, t_{1}+\frac{1}{2(1+\alpha)} k_{+}^{-(1+\alpha)}\right]$, we have in particular that $|v(x, t)| \leq K|x| \leq \frac{K^{2}}{4 \varepsilon}+\varepsilon|x|^{2}$ for any $\varepsilon>0$, for $|x| \leq 2^{-\frac{1}{1+\alpha}} k_{+}^{-1}$ when $t=t_{1}$ and for $|x|=2^{-\frac{1}{1+\alpha}} k_{+}^{-1}$ when $t_{1} \leq t \leq t_{1}+\frac{1}{2(1+\alpha)} k_{+}^{-(1+\alpha)}$. Sub and super solutions for equation (8.3) are then given by $v_{ \pm}^{(\varepsilon)}= \pm\left(\frac{K^{2}}{4 \varepsilon}+\varepsilon|x|^{2}+2 \varepsilon\left(\Lambda n+2^{-\frac{1}{1+\alpha}} k_{+}^{-1} B\right)\left(t-t_{1}\right)\right)$, and so by the comparison principle we have $\left|v\left(x_{1}, t\right)\right| \leq \frac{K^{2}}{4 \varepsilon}+2 \varepsilon\left(\Lambda n+2^{-\frac{1}{1+\alpha}} k_{+}^{-1} B\right)\left(t-t_{1}\right)$. Optimizing over $\varepsilon$ for each $t$ gives $\left|v\left(x_{1}, t\right)\right| \leq \sqrt{2\left(\Lambda n+2^{-\frac{1}{1+\alpha}} k_{+}^{-1} B\right) K^{2}\left(t-t_{1}\right)}$. The continuity of the unit normal $\nu$ follows since it is a smooth function of the gradient.

We can now deduce Hölder continuity of the speed $F$ :

Lemma 8.4. For $X$ as in Theorem 8.1, there exist $\gamma \in(0,1), C$ and d depending only on $r_{+}\left(M_{0}\right), r_{-}\left(M_{\tau}\right)$ and $\inf _{M_{0}} F$ such that

$$
|F(x, t)-F(y, s)| \leq C\left(|X(x, t)-X(y, s)|^{\gamma}+|t-s|^{\gamma / 2}\right)
$$

for all $(x, t)$ and $(y, s)$ in $M \times[\tau / 4, \tau]$ with $|X(x, t)-X(y, s)|+\sqrt{|t-s|}<d$.

Proof. As before we write the evolving hypersurface locally as an evolving graph satisfying equation (8.2). Differentiating with respect to time, we find that the function $v=\frac{\partial u}{\partial t}$ again satisfies equation (8.3). The expression (8.4) for $a^{i j}$, together with bounds on $D u$ and $F$ and equations (4.3) and (4.4), imply that there exist $\lambda$ and $\Lambda$ such that $\lambda\|\xi\|^{2} \leq a^{i j} \xi_{i} \xi_{j} \leq \Lambda\|\xi\|^{2}$ for all $\xi$. The bounds on $D u$ and $D^{2} u$ also imply that $\left|b^{i}\right| \leq B$. The function $\tilde{v}(z, t)=R^{-1} v\left(R z, t_{1}+(1+t) R^{2} t\right)$ with $R=\min \left\{1,2^{-\frac{1}{1+\alpha}} k_{+}^{-1}, \frac{1}{\sqrt{2(1+\alpha)}} k_{+}^{-\frac{1+\alpha}{2}}\right\}$ gives a solution of equation (8.3) on $Q_{1}$ 
with $b$ replaced by $R b$ (hence $|b| \leq B$ ) still holds), and so Theorem 7.1 applies. Rescaling back we have the estimate

$$
\underset{Q_{r}\left(0, t_{1}+R^{2}\right)}{\operatorname{Osc}} v \leq K\left(\frac{r}{R}\right)^{\gamma} \underset{Q_{R}\left(0, t_{1}+R^{2}\right)}{\operatorname{Osc}} v
$$

for $0<r<R$. Finally, since $\frac{\partial u}{\partial t}$ and $D u$ are Hölder continuous, so is $F$ by equation (8.2).

Now we complete the proof of Theorem 8.1. Choose any $\left(x_{1}, t_{1}\right)$ with $t_{1} \in$ $[\tau / 4, \tau]$. We first rescale to ensure that $F\left(x_{1}, t_{1}\right)=1$, by setting $Y(x, t)=$ $F\left(x_{1}, t_{1}\right)^{1 / \alpha} X\left(x, t_{1}+F\left(x_{1}, t_{1}\right)^{-\frac{1+\alpha}{\alpha}} t\right)$ and noting that $Y$ is again a solution of (4.1). We write the evolving hypersurface $Y_{t}(M)$ locally near $\left(x_{1}, 0\right)$ as a graph evolving according to equation (8.2), and let $v=\frac{\partial u}{\partial z^{i}}$ for some $i \in\{1, \ldots, n\}$, so that $v$ evolves according to equation (8.3). The expressions (8.4) and (8.5), together with the Hölder continuity of $D u$ and $F$, imply that on small enough regions we can make $|D u|$ as small as desired (in particular less than 1) and $F$ as close as desired to 1. By the expression (8.4) for $a^{i j}$, and the bounds (4.3) and (4.4), on small regions we have $\lambda\|\xi\|^{2} \leq a^{i j} \xi_{i} \xi_{j} \leq \Lambda\|\xi\|^{2}$, where $\lambda$ and $\Lambda$ depend only on $n, \alpha$ and $\mu$. Similarly, since $F$ is close to 1 we have bounds above and below on principal curvatures from Lemma 2.2, and hence bounds $|D v| \leq B$ and $\left|b^{i} D_{i} v\right| \leq A$, where $A$ depends only on $n, \alpha$ and $\mu$. Let $\sigma$ be given by Theorem 7.3 with these values of $\lambda, \Lambda, A$ and $B$. Finally, by (4.3) and (4.4) we can choose $\delta_{2}$ sufficiently small (depending on $n, \alpha, \mu$ and $\sigma)$ to ensure that $(\alpha-\sigma / 4) I \leq \dot{F}(A) \leq(\alpha+\sigma / 4) I$ whenever $F(A)=1$ and $\|\AA\|^{2} \leq \delta_{2} H^{2}$. It follows that on small enough regions we have $\left|a^{i j}(x, t)-a^{i j}(y, s)\right|<\sigma$.

Now let $\tilde{v}(z, t)=R^{-1} v\left(R z, R^{2} t\right)$ for $R$ to be chosen. Then $v$ satisfies equation (8.3) on $Q_{1}$ (for $R$ sufficiently small) with $b^{i}$ replaced by $R b^{i}$, and hence for sufficiently small $R$ (depending on $n, \alpha, \mu, r_{ \pm}$and $\inf _{M_{0}} F$ ) we can apply Theorem 7.3 to deduce $C^{1, \gamma}$ estimates on $\tilde{v}$. Translating back to $v$ we deduce $C^{2, \gamma}$ bounds on $u$. This implies Hölder continuity of the second fundamental form of the solution $Y_{t}$, and again scaling back gives Hölder continuity of the second fundamental form of $X_{t}$, as claimed.

\section{Higher REGULARITY}

The higher regularity for solutions of equation (4.1) now follows from Schauder estimates:

Proposition 9.1. For each $k \in \mathbb{N}$ there exists $C_{k}$ depending on $t_{0}, n, \alpha, F, r_{ \pm}$ and $\inf _{M_{0}} F$ such that for any solution $X: M \times\left[0, t_{0}\right] \rightarrow \mathbb{R}^{n+1}$ of equation (4.1) satisfying $\|\AA\|^{2}<\min \left\{\delta_{0}, \delta_{2}\right\} H^{2},\left\|\nabla^{k} A\right\| \leq C_{k}$ on $M \times\left[t_{0} / 2, t_{0}\right]$.

Proof. We again work with the local graph parametrization used in the previous section, around some point $\left(x_{1}, t_{1}\right)$. Since $\nabla^{k} A$ is controlled once the derivatives up to order $(k+2)$ are controlled when the hypersurface is written locally as a graph, it suffices to obtain bounds on $D^{k} u$ for the corresponding solutions of equation (8.2).

By Theorem 8.1, the function $u$ is $C^{2, \gamma}$, with Hölder bounds on second derivatives depending on $n, \alpha, \mu, r_{ \pm}, \inf _{M_{0}} F$, and elapsed time. In particular this implies that the coefficients of equation (8.3) are Hölder continuous, and therefore by Theorem 7.2 we deduce $C^{2, \gamma}$ bounds on $v=\frac{\partial u}{\partial z^{i}}$, and hence $C^{3, \gamma}$ bounds on $u$. The higher regularity follows by induction, by considering the evolution equations satisfied by 
higher spatial derivatives of $u$ : The Hölder continuity of $m$ th spatial derivatives of $u$ allows Theorem 7.2 to be applied to the evolution equation for order $m-1$ spatial derivatives, yielding Hölder continuity of order $m+1$ derivatives. By induction this yields bounds on all spatial derivatives, and by the evolution equation this also implies bounds on arbitrary spatial and time derivatives of $u$ also.

\section{Convergence to a point}

Proposition 10.1. Let $M_{0}=X_{0}(M)$ be a smooth, uniformly convex, compact hypersurface with $\|\AA\|^{2}<\min \left\{\delta_{0}, \delta_{2}\right\} H^{2}$. Then the maximally defined solution $X: M \times[0, T) \rightarrow \mathbb{R}^{n+1}$ of equation (4.1) with initial data $X_{0}$ is $C^{\infty}$, each hypersurface $M_{t}=X_{t}(M)$ is strictly locally convex, and there exists $p \in \mathbb{R}^{n+1}$ such that $X_{t}(M) \rightarrow p \in \mathbb{R}^{n+1}$ uniformly as $t \rightarrow T$.

Proof. Suppose the inradius does not approach zero as the final time is approached. At $t=0$ the speed $F$ has a positive lower bound since $M_{0}$ is uniformly convex, and this is maintained as long as the solution exists (see Remark 5.2). Proposition 6.1 implies that $F$ is bounded since the inradius does not approach zero, and the pinching estimate of Theorem 5.1 and Lemma 2.2 then imply bounds above and below on principal curvatures. Proposition 9.1 then provides bounds on all higher derivatives of curvature. In this case the solution can be continued to a larger time interval, by the argument of [H1, Theorem 8.1]. This would contradict the maximality of the interval of existence, so the inradius must approach zero as $t \rightarrow T$. The circumradius does also, by [A1, Lemma 5.4].

\section{IMPROVING PINCHING}

Now we prove that the bounds on the pinching ratio can be improved to show that the pinching ratio is close to one when the curvature becomes large. For $\alpha>1$ this can be done using the maximum principle, rather than the more involved integral estimates and iteration techniques which are required to prove the corresponding estimates for mean curvature flow in [H1].

The result is the following:

Theorem 11.1. Let $\delta>0$ be as in Theorem 5.1, Let $M_{0}=X_{0}(M)$ be a smooth, compact, strictly convex hypersurface for which $\|\AA\|^{2}<\sigma_{0} H^{2}$ with $\sigma_{0} \in(0, \delta)$. Let $\mathfrak{h}=\sup _{M_{0}} H$, and let $X: M \times[0, T) \rightarrow \mathbb{R}^{n+1}$ be the solution of 4.11). Then there exists $\lambda>0$ such that for any $t \geq 0$, the second fundamental form $A$ of $M_{t}$ lies in the set

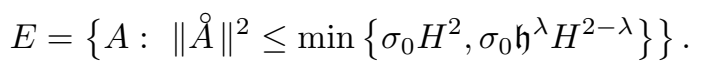

Proof. At $t=0$ we have $H \leq \mathfrak{h}$, and so $\|\AA\|^{2}<\sigma_{0} H^{2}=\min \left\{\sigma_{0} H^{2}, \sigma_{0} \mathfrak{h}^{\lambda} H^{2-\lambda}\right\}$. We have already proved that the condition $\|\AA\|^{2} \leq \sigma_{0} H^{2}$ is preserved. It remains to prove that for suitable $\lambda>0$ the quantity $\bar{Z}=\|\AA\|^{2}-\sigma_{0} \mathfrak{h}^{\lambda} H^{2-\lambda}$ cannot attain a new zero maximum at a point where $H \geq \mathfrak{h}$. At such a point we let $\sigma=\sigma_{0}\left(\frac{\mathfrak{h}}{H}\right)^{\lambda} \leq \sigma_{0}$, and note that $Z=0, \mathcal{L} Z \leq 0$ and $\partial_{t} Z \geq 0$. The latter are 
related by the evolution equation for $Z$ :

$$
\begin{aligned}
\frac{\partial Z}{\partial t}= & \mathcal{L} Z+2\left(h^{i j}-\left(\frac{1}{n}+\sigma\right) H g^{i j}\right) \ddot{F}^{k l, r s} \nabla_{i} h_{k l} \nabla_{j} h_{r s} \\
& -2 \dot{F}^{i j}\left(\nabla_{i} h_{k l} \nabla_{j} h^{k l}-\frac{1}{n} \nabla_{i} H \nabla_{j} H\right)+2 \sigma \dot{F}^{i j} \nabla_{i} H \nabla_{j} H \\
& +\lambda \sigma H g^{i j} \ddot{F}\left(\nabla_{i} h, \nabla_{j} h\right)-\sigma \lambda(3-\lambda) \dot{F}^{i j} \nabla_{i} H \nabla_{j} H+2 \dot{F}^{i j} h_{i m} h^{m}{ }_{j} Z \\
& +\lambda \sigma \dot{F}^{i j} h_{i m} h^{m}{ }_{j} H^{2}-\frac{2(\alpha-1) F}{n}\left(n C-(1+n \sigma) H\|A\|^{2}+\frac{n \lambda \sigma}{2} H\|A\|^{2}\right) .
\end{aligned}
$$

The terms which do not involve $\lambda$ can be estimated exactly as in the proof of Theorem 5.1. For the remaining terms we have the following straightforward estimates: Choosing $\lambda \leq 3$ we discard the third term on the second line. The preceding term we estimate by

$$
\lambda \sigma H \ddot{F}\left(\nabla_{i} h, \nabla_{i} h\right) \leq \lambda \sigma H^{\alpha-1} M_{2}(\sigma)\|\nabla A\|^{2} .
$$

Thus all of the terms involving gradients of curvature can be estimated by $H^{\alpha-1}\|\nabla A\|^{2}$ times

$$
-\frac{4(n-1)}{3 n} M_{1}(\sigma)+2 \sqrt{\sigma(1+n \sigma)} M_{2}(\sigma)+\frac{2(n+2)}{3} \sigma\left(\alpha+\sqrt{\sigma} M_{2}(\sigma)\right)+\lambda \sigma M_{2}(\sigma) .
$$

Since $\sigma \leq \sigma_{0}<\delta$, the first three terms have a strictly negative sum, and so the result is nonpositive provided $\lambda$ is sufficiently small depending on $\sigma_{0}$.

The terms in the last line of equation (11.1) can be estimated as follows: We discard the negative term in the last bracket, and estimate the others using Lemma 2.3. The preceding term is bounded as follows: Since the principal curvatures are nonnegative we have $h_{i m} h^{m}{ }_{j} \leq H h_{i j}$, and so

$$
\lambda \sigma \dot{F}^{i j} h_{i m} h^{m}{ }_{j} H^{2} \leq \lambda \sigma \dot{F}^{i j} h_{i j} H^{3}=\lambda \alpha \sigma F H^{3} .
$$

This gives the following bound for the last line of (11.1):

$$
\left(\alpha \lambda \sigma-\frac{2}{n}(\alpha-1) \sigma(1+n \sigma)(1-\sqrt{n(n-1) \sigma})\right) F H^{3},
$$

which is strictly negative for $\lambda>0$ sufficiently small depending only on $\sigma_{0}$ and $\alpha$.

Corollary 11.2. If $X$ is a solution of equation (4.1) as in Theorem 11.1, then for any $\varepsilon>0$ there exists a constant $C_{1}(\varepsilon) \geq 0$ such that $\kappa_{n} \leq(1+\varepsilon) \kappa_{1}+C_{1}(\varepsilon)$.

Proof. By Theorem 11.1 we have

$$
\begin{aligned}
\kappa_{n}-\kappa_{1} & \leq \sqrt{n}\|\stackrel{\AA}{A}\| \\
& \leq \sqrt{n \sigma_{0}} \mathfrak{h}(H / \mathfrak{h})^{1-\lambda / 2} \\
& \leq \sqrt{n \sigma_{0}} \mathfrak{h}\left(\frac{1}{p} a^{-p}+\frac{1}{q} a^{q}(H / \mathfrak{h})^{q(1-\lambda / 2)}\right)
\end{aligned}
$$

for any $a>0$ and $1 / p+1 / q=1$, by Young's inequality. Choosing $q(1-\lambda / 2)=1$ gives

$$
\kappa_{n}-\kappa_{1} \leq \sqrt{n \sigma_{0}}\left[\frac{2-\lambda}{\lambda} a^{2 /(2-\lambda)} H+\frac{\lambda}{2} \mathfrak{h} a^{-2 / \lambda}\right] \leq \varepsilon \kappa_{1}+C_{1}(\varepsilon)
$$


for any $\varepsilon>0$, where we used Lemma 2.2 to estimate $H$ in terms of $\kappa_{1}$, and chose $a$ to satisfy $\sqrt{n \sigma_{0}} \frac{2-\lambda}{\lambda}=\left(1-\sqrt{n(n-1) \sigma_{0}}\right) \varepsilon$. Accordingly $C_{1}(\varepsilon)$ is given by

$$
C_{1}(\varepsilon)=\frac{\lambda \mathfrak{h}}{2} \sqrt{n \sigma_{0}}\left(\frac{2 \varepsilon\left(1-\sqrt{n(n-1) \sigma_{0}}\right)}{(2-\lambda) n^{3 / 2} \sqrt{\sigma_{0}}}\right)^{-\frac{2-\lambda}{\lambda}} .
$$

\section{LOWER SPEED BOUNDS}

The results of Theorem 3.1 and Corollary 11.2 imply that the evolving hypersurfaces are close to spheres when their diameter is small. In this section we use this to deduce lower bounds on the speed. Our argument here is quite general and does not require any assumptions on the speed except for homogeneity and parabolicity.

Proposition 12.1. Let $X: M \times[0, T)$ be a smooth, strictly convex solution of a flow of the form (4.1) which contracts to a point as $t \rightarrow T$, and such that the ratio of the circumradius to the inradius approaches 1 as $t \rightarrow T$. Then there exists $t_{-}<T$ and $C>0$ such that $F(z, t) \geq C(T-t)^{-\frac{\alpha}{1+\alpha}}$ for all $z \in \bar{M}$ and $t \in\left[t_{-}, T\right)$.

Proof. We begin with the following observation, originally due to Smoczyk S4, Proposition 4] for the mean curvature flow and proved here by a different method:

Lemma 12.2. If $M_{t_{0}}$ encloses $p \in \mathbb{R}^{n+1}$, then $\langle X-p, \nu\rangle+(1+\alpha)\left(t-t_{0}\right) F \geq 0$ for $t_{0} \leq t<T$.

Proof of the lemma. The homogeneity of $F$ implies that

$$
X_{\lambda}(z, t)=\lambda\left(X\left(z, \lambda^{-(1+\alpha)}\left(t-t_{0}\right)\right)-p\right)+p
$$

is again a solution of the same flow, for any $\lambda>0$ and $p \in \mathbb{R}^{n+1}$. When $t=t_{0}$ this amounts to a rescaling about the point $p$, so if $M_{t_{0}}$ encloses $p$, then $X_{\lambda}\left(M, t_{0}\right)$ encloses $M_{t_{0}}$ for any $\lambda>1$. By the comparison principle, $X_{\lambda}(\bar{M}, t)$ encloses $M(t)$ for each $t \geq t_{0}$ and $\lambda>1$, and we deduce that

$$
\left\langle\left.\frac{\partial}{\partial \lambda} X_{\lambda}(z, t)\right|_{\lambda=1}, \nu(z, t)\right\rangle=\left.\frac{\partial}{\partial \lambda}\left\langle X_{\lambda}(z, t), \nu(z, t)\right\rangle\right|_{\lambda=1} \geq 0
$$

for all $z$ and $t \geq t_{0}$. Computing the left-hand side explicitly, we find

$$
0 \leq\left\langle X-p+(1+\alpha)\left(t-t_{0}\right) F \nu, \nu\right\rangle=\langle X-p, \nu\rangle+(1+\alpha)\left(t-t_{0}\right) F .
$$

Proof of the proposition, continued. Choose $t_{1}$ close enough to $T$ to ensure $r_{+}\left(M_{t}\right)$ $\leq(1+\varepsilon) r_{-}\left(M_{t}\right)$ for all $t \in\left[t_{1}, T\right)$, where $\varepsilon>0$ is such that $(1+2 \varepsilon)^{1+\alpha}<5 / 4$. Fix $t_{0} \in\left[t_{1}, T\right)$, and write $r_{-}=r_{-}\left(M_{t_{0}}\right)$. Choose $q \in \mathbb{R}^{n+1}$ to be the incentre of $M_{t_{0}}$, so that $B_{r_{-}}(q)$ is enclosed by $M_{t_{0}}$, and $B_{(1+2 \varepsilon) r_{-}}(q)$ encloses $M_{t_{0}}$. By the comparison principle, $B_{r(t)}(q)$ is enclosed by $M_{t}$ for $t \geq t_{0}$, where $r(t)=$ $\left(r_{-}^{1+\alpha}-(1+\alpha)\left(t-t_{0}\right)\right)^{1 /(1+\alpha)}$, and $B_{R(t)}(q)$ encloses $M_{t}$ for $t \geq t_{0}$, where $R(t)=$ $\left(\left((1+2 \varepsilon) r_{-}\right)^{1+\alpha}-(1+\alpha)\left(t-t_{0}\right)\right)^{1 /(1+\alpha)}$. In particular this implies $T \geq t_{0}+\frac{r_{-}^{1+\alpha}}{1+\alpha}$. 
Fix $z \in \bar{M}$, and let $t=t_{0}+\frac{3 r_{-}^{1+\alpha}}{4(1+\alpha)}$. Choose $p$ to be the point in $M_{t_{0}}$ which maximizes $\langle p, \nu(z, t)\rangle$, and apply Lemma 12.2, This gives

$$
F(z, t) \geq \frac{\langle p-X(z, t), \nu(z, t)\rangle}{(1+\alpha)\left(t-t_{0}\right)} \geq \frac{4\left(r_{-}-R(t)\right)}{3 r_{-}^{1+\alpha}} .
$$

The estimates above give $R(t)=r_{-}\left((1+2 \varepsilon)^{1+\alpha}-3 / 4\right)^{1 /(1+\alpha)} \leq 2^{-1 /(1+\alpha)} r_{-}$by our choice of $\varepsilon$. Also note that $T-t \geq \frac{r_{-}^{1+\alpha}}{4(1+\alpha)}$. These imply

$$
F(z, t) \geq \frac{4\left(1-2^{-1 /(1+\alpha)}\right)}{3(4(1+\alpha)(T-t))^{\alpha /(1+\alpha)}}=C(T-t)^{-\frac{\alpha}{1+\alpha}},
$$

as required, for all $z \in \bar{M}$ and all $t \geq t_{-}=t_{1}+\frac{3 r_{-}\left(t_{1}\right)^{1+\alpha}}{4(1+\alpha)}$.

\section{The CONVERGENCE THEOREM}

Proof of Theorem 4.2. The existence until the solution converges to a point $p$ was proved in Proposition 10.1. We next prove Hausdorff convergence of the rescaled hypersurfaces $\tilde{M}_{t}=\tilde{X}_{t}(M)$ to the unit sphere: By Corollary 11.2 and Theorem 3.1. for any $\rho>0$ there exists $C_{2}(\rho)$ such that $r_{+}\left(M_{t}\right) \leq(1+\rho) r_{-}\left(M_{t}\right)$ whenever $r_{+}(M) \leq C_{2}(\rho)$. Since $M_{t}$ converges to a point we have $r_{+}\left(M_{t}\right) \rightarrow 0$ as $t \rightarrow T$, so for each $\rho>0$ there exists $t(\rho)<T$ such that $r_{+}\left(M_{t}\right) \leq(1+\rho) r_{-}\left(M_{t}\right)$ for $t(\rho) \leq t<T$, and since $M_{t}$ converges to $p, p$ is enclosed by $M_{t}$ for each $t$. We can relate $r_{ \pm}\left(M_{t}\right)$ to $T-t$. The time of existence is no less than that of the sphere of radius $r_{-}\left(M_{t}\right)$, and no greater than that of the sphere of radius $r_{+}\left(M_{t}\right)$, so

$$
\frac{r_{-}\left(M_{t}\right)^{1+\alpha}}{1+\alpha} \leq T-t \leq \frac{r_{+}\left(M_{t}\right)^{1+\alpha}}{1+\alpha} \leq \frac{\left((1+\rho) r_{-}\left(M_{t}\right)\right)^{1+\alpha}}{1+\alpha}
$$

for $t \geq t(\rho)$. We can also control the distance from $p$ to the centre $p_{t}$ of the insphere of $M_{t}: p$ is enclosed by $M_{t^{\prime}}$, which is enclosed by the sphere of radius $\left(r_{+}\left(M_{t}\right)^{1+\alpha}-(1+\alpha)\left(t^{\prime}-t\right)\right)^{1 /(1+\alpha)}$ about $p_{t}$ for $t \leq t^{\prime}<T$. Taking $t^{\prime} \rightarrow T$ gives $\left|p-p_{t}\right| \leq\left(\left(r_{+}\left(M_{t}\right)^{1+\alpha}-(1+\alpha)(T-t)\right)^{1 /(1+\alpha)} \leq\left(r_{+}\left(M_{t}\right)^{1+\alpha}-r_{-}\left(M_{t}\right)^{1+\alpha}\right)^{1 /(1+\alpha)}\right.$. This implies that

$$
\frac{\left|p_{t}-p\right|}{((1+\alpha)(T-t))^{1 /(1+\alpha)}} \leq\left((1+\rho)^{1+\alpha}-1\right)^{1 /(1+\alpha)}
$$

for $t>t(\rho)$. The estimates (13.1) and (13.2) amount to Hausdorff convergence of $\tilde{M}_{t}$ to the unit sphere.

Next we observe that by Proposition 12.1 for $t \geq t_{-}$we have $F \geq C(T-t)^{-\frac{\alpha}{1+\alpha}}$, and hence by the pinching estimate of Theorem 5.1 and Lemma 2.2 we have $\kappa_{\min } \geq$ $(T-t)^{-\frac{1}{1+\alpha}}$.

We deduce an upper curvature bound from Proposition 12.1, using scaling to deduce the correct dependence on the remaining time. Fix $t_{*} \in[T / 2, T)$, and consider rescaled solutions defined by

$$
\tilde{X}^{\left(t_{*}\right)}(p, t)=\frac{X\left(p, t_{*}+\left(T-t_{*}\right) t\right)}{\left(T-t_{*}\right)^{\frac{1}{1+\alpha}}} .
$$

For any $t^{*} \in[T / 2, T), \tilde{X}^{\left(t_{*}\right)}$ is a solution of equation (4.1) on $M \times[-1,0]$. Given $\rho>0$, if $T-t_{*}<(T-t(\rho)) / 2$, then by (13.1) we have $r_{-}\left(\tilde{X}_{t}^{\left(t_{*}\right)}\right) \geq \frac{(1+\alpha)^{\frac{1}{1+\alpha}}}{1+\rho}$ and 
$r_{+}\left(\tilde{X}_{t}^{\left(t_{*}\right)}\right) \leq(1+\rho)(2(1+\alpha))^{\frac{1}{1+\alpha}}$, for $-1 \leq t \leq 0$. It follows from Proposition 12.1 that the rescaled solutions have $F(x, 0) \leq C$ independent of $t_{*}$, and hence the unrescaled solutions have $F \leq C(T-t)^{-\frac{\alpha}{1+\alpha}}$.

Estimates on higher derivatives of curvature also follow: The solutions $\tilde{X}^{\left(t_{*}\right)}$ defined above have bounds on curvature in $C^{k}$ for any $k$ on $M \times[-1 / 2,0]$, by Proposition 9.1. It follows that $\left\|\nabla^{(k)} \mathcal{W}\right\| \leq C_{k}(T-t)^{-\frac{\alpha(1+k)}{1+\alpha}}$ for each $k$, and the rescaled hypersurfaces $\frac{M_{t}-p}{((1+\alpha)(T-t))^{\frac{1}{1+\alpha}}}$ have uniform bounds on curvature and all higher derivatives.

$C^{\infty}$ convergence of the rescaled embeddings now follows: The pinching estimate of Theorem 11.1 implies that (for $t$ close enough to $T$ ) the rescaled maps satisfy $\|\AA\|^{2} \leq C(T-t)^{\frac{\lambda}{1+\alpha}}$. By interpolation we also have $\|\nabla \AA\| \leq C(T-t)^{\beta}$ for some $\beta>0$, and hence by Lemma 2.1] $\|\nabla A\| \leq C(T-t)^{\beta}$. Then interpolation again gives that $\left\|\nabla^{(k)} A\right\| \leq C_{k}(T-t)^{\beta_{k}}$ for every $k$, where $\beta_{k}>0$. One can define a normalized flow as in [H1, Section 9]. The time variable in the normalized flow is $\tau=\log (T-t)$, so we have exponential decay of all derivatives of the second fundamental form for the normalized flow, and the argument in [H1, Section 10] can be applied.

Remark. A similar result to Theorem 4.2 also holds in the case $\alpha=1$ : By a slight modification of the proof of Theorem 5.1, an essentially arbitrary smooth, homogeneous degree one speed $F$ preserves sufficiently strong pinching of a convex hypersurface, with pinching ratio determined by $n$ and a bound for the second derivatives of $F$. The argument given in Section 8 applies with minor changes to prove Hölder continuity of the second fundamental form, and higher regularity follows by Schauder estimates. Convergence of the rescaled hypersurfaces then follows by the argument given in A1. We note that a lower bound on $F$ follows in this case from the Krylov-Safonov Harnack inequality, so this situation does not need the geometric result of Section 3.

\section{REFERENCES}

[A] Roberta Alessandroni, Evolution of hypersurfaces by curvature functions, Ph.D. Thesis, Università degli studi di Roma "Tor Vergata", 2008, http://dspace.uniroma2.it/dspace/ handle/2108/661.

[AS] Roberta Alessandroni and Carlo Sinestrari, Evolution of hypersurfaces by powers of the scalar curvature, Ann. Sc. Norm. Super. Pisa Cl. Sci. (5) 9 (2010), 541-571. MR.2722655

[A1] Ben Andrews, Contraction of convex hypersurfaces in Euclidean space, Calc. Var. Partial Differential Equations 2 (1994), no. 2, 151-171. MR.1385524 (97b:53012)

[A2] _ Gauss curvature flow: the fate of the rolling stones, Invent. Math. 138 (1999), no. 1, 151-161. MR.1714339 (2000i:53097)

[A3] , Pinching estimates and motion of hypersurfaces by curvature functions, J. Reine Angew. Math. 608 (2007), 17-33. MR2339467(2008i:53087)

[A4] _ Moving surfaces by non-concave curvature functions, Calc. Var. Partial Differential Equations 39 (2010), 649-657. MR2729317

[CRS] Esther Cabezas-Rivas and Carlo Sinestrari, Volume-preserving flow by powers of the $m$ th mean curvature, Calc. Var. Partial Differential Equations 38 (2010), 441-469. MR2647128 (2011g;53141)

[Ca] Luis A. Caffarelli, Interior a priori estimates for solutions of fully nonlinear equations, Ann. of Math. (2) 130 (1989), no. 1, 189-213. MR.1005611 (90i:35046)

[Ch1] Bennett Chow, Deforming convex hypersurfaces by the nth root of the Gaussian curvature, J. Differential Geom. 22 (1985), no. 1, 117-138. MR826427 (87f:58155) 
[Ch2] - Deforming convex hypersurfaces by the square root of the scalar curvature, Invent. Math. 87 (1987), no. 1, 63-82. MR862712 (88a:58204)

[Co] Heinz Otto Cordes, Über die erste Randwertaufgabe bei quasilinearen Differentialgleichungen zweiter Ordnung in mehr als zwei Variablen, Math. Ann. 131 (1956), 278-312 (German). MR0091400 (19,961e)

[E] Lawrence C. Evans, Classical solutions of fully nonlinear, convex, second-order elliptic equations, Comm. Pure Appl. Math. 35 (1982), no. 3, 333-363. MR649348 (83g:35038)

[G] Georges Glaeser, Fonctions composées différentiables, Ann. of Math. (2) 77 (1963), 193-209 (French). MR0143058 (26 \#624)

[H1] Gerhard Huisken, Flow by mean curvature of convex surfaces into spheres, J. Differential Geom. 20 (1984), no. 1, 237-266. MR772132 (86j:53097)

[H2] _ The volume preserving mean curvature flow, J. reine angew. Math. 382 (1987), 35-48. MR921165 (89d:53015)

[K] N. V. Krylov, Boundedly inhomogeneous elliptic and parabolic equations, Izv. Akad. Nauk SSSR Ser. Mat. 46 (1982), no. 3, 487-523, 670 (Russian). MR661144(84a:35091)

[KS] N. V. Krylov and M. V. Safonov, A property of the solutions of parabolic equations with measurable coefficients, Izv. Akad. Nauk SSSR Ser. Mat. 44 (1980), no. 1, 161-175, 239 (Russian). MR.563790 (83c:35059)

[L] Gary M. Lieberman, Second order parabolic differential equations, World Scientific Publishing Co. Inc., River Edge, NJ, 1996. MR1465184 (98k:35003)

$[\mathrm{N}]$ L. Nirenberg, On a generalization of quasi-conformal mappings and its application to elliptic partial differential equations, Contributions to the theory of partial differential equations, Annals of Mathematics Studies, no. 33, Princeton University Press, Princeton, N. J., 1954, pp. 95-100. MR0066532(16,592a)

[S1] Rolf Schneider, Convex bodies: the Brunn-Minkowski theory, Encyclopedia of Mathematics and its Applications, vol. 44, Cambridge University Press, Cambridge, 1993. MR1216521 (94d:52007)

[S2] Oliver C. Schnürer, Surfaces contracting with speed $|A|^{2}$, J. Differential Geom. 71 (2005), no. 3, 347-363. MR2198805 (2006i:53099)

[S3] Felix Schulze, Convexity estimates for flows by powers of the mean curvature, Ann. Sc. Norm. Super. Pisa Cl. Sci. (5) 5 (2006), no. 2, 261-277. MR2244700 (2007b:53138)

[S4] Knut Smoczyk, Starshaped hypersurfaces and the mean curvature flow, Manuscripta Math. 95 (1998), no. 2, 225-236. MR1603325 (99c:53033)

[T] Kaising Tso, Deforming a hypersurface by its Gauss-Kronecker curvature, Comm. Pure Appl. Math. 38 (1985), no. 6, 867-882. MR812353(87e:53009)

Centre for Mathematics and its Applications, Australian National University, ACT 0200 Australia

E-mail address: Ben.Andrews@maths.anu.edu.au

Institute of Mathematics and Applied Statistics, University of Wollongong, WolLONGONG, NSW 2522, Australia

E-mail address: jamesm@uow.edu.au 